\title{
The Effects of Mergers in Open Auction Markets*
}

\author{
KeITH WAEHRER \\ U.S. Department of Justice, Suite 10-000 \\ 600 E Street, N.W., Washington, DC 20212, U.S.A. \\ PHONE: 202-307-6365, EMAIL: keith.waehrer@usdoj.gov \\ MARtin K. PerRy \\ Department of Economics, Rutgers University \\ New Brunswick, NJ 08901 U.S.A. \\ PHONE: 732-932-7572, EMAIL: martinperry@worldnet.att.net
}

Current version: September 24, 2001

First version: May 22, 1998

\begin{abstract}
The buyer solicits bids from suppliers with different cost distributions defined by their capacities. The expected market share of each supplier is the ratio of its capacity to the industry capacity. The buyer's optimal reserve price declines with increases in the concentration of the industry. The lower reserve price can partially or fully offset the price effects of a merger. However, a merger still reduces the buyer's welfare because there is an increased probability of internal production at a higher cost. The lower reserve price can also undermine the incentive for larger suppliers to merge and result in stable industry structures for which no further mergers would be profitable.
\end{abstract}

\footnotetext{
* Any views expressed in this paper are not purported to reflect those of the United States Department of Justice. This paper benefited from the comments of Roberto Burguet, Robert Bradford, Serdar Dalkir, Luke Froeb, Ian Gale, Patrick Greenlee, Preston McAfee, Rich McLean, Mike Rothkopf, Jozsef Sakovics, Guofu Tan, and Charles Thomas.
} 


\section{The Effects of Mergers in Open Auction Markets}

The effects of mergers have been examined in a number of papers using the traditional Nash-Cournot model with a homogeneous product, but allowing firms to have different capacities. ${ }^{1}$ Several recent papers have examined mergers in auction markets with firms having different cost distributions. ${ }^{2}$ In this paper, we investigate the effects of mergers in an asymmetric auction model of procurement. The buyer employs an open auction with a reserve price to purchase an input from suppliers having private information about their cost of producing the input. We characterize a family of cost distributions which has a natural interpretation for the size of the suppliers. Using this model, we examine the traditional merger questions. Does a merger increase the price and reduce the welfare of the buyer? Do the suppliers have an incentive to merge? Does a merger reduce total welfare? The answers to these questions in this model will have some similarities and some differences from those obtained using the Nash-Cournot model.

Antitrust enforcement agencies such as the Department of Justice and the Federal Trade Commission and private plaintiffs must frequently evaluate mergers in industrial product markets governed by an auction. The winner-take-all characteristic of auction models can lead to misinterpretations when used to analyze the competitive effects of a merger. One naive argument is that all the suppliers have an equal chance of winning each auction and thus the industry should be treated as a symmetric oligopoly for assessing concentration. Another naive argument is that each auction is a separate market and will inherently have only a single supplier. Both of these arguments distort the competitive conditions and are designed to limit the scope of enforcement. Auction markets also provide an opportunity for implausible defenses. For example, the merging suppliers may argue that they rarely

\footnotetext{
${ }^{1}$ Perry and Porter (1985) consider the incentive to merge in a Nash-Cournot model with a homogeneous product. Farrell and Shapiro (1990a and 1990b) examine a general version of the capacity model used by Perry and Porter (1985), and focus on the welfare effects of mergers. With the linear version of this capacity model, McAfee and Williams (1992) characterize the profitable mergers between two firms that would increase welfare. Werden (1991) also reexamines the linear model in order to illustrate the relevance of the HerfindahlHirschman Index (HHI) in assessing the price effects of mergers. Deneckere and Davidson (1985) examine mergers in a market with differentiated products where multibrand firms are comparable to suppliers with a larger capacity.

${ }^{2}$ Waehrer (1997) examines mergers in both asymmetric first-price and second-price auction markets. Dalkir, Logan, and Masson (2000) and Tschantz, Crooke, and Froeb (2000) examine mergers in asymmetric first-price auctions using simulated equilibrium bidding strategies. Thomas (1998) examines mergers in asymmetric first-price auctions by deriving equilibrium bidding strategies for the binomial cost distribution. Brannman and Froeb (2000) and Froeb, Tschantz, and Crooke (1998) examine mergers in asymmetric second-price auctions with the extreme value cost distribution. There is also a related literature on collusion in auction markets. For example, see DeBrock and Smith (1983), Graham and Marshall (1987), Von Ungern-Sternberg (1988), Mailath and Zemsky (1991), McAfee and McMillan (1992), and McAfee (1994). More recently, Mares (2000a and 2000b) examines mergers in asymmetric common-value auctions.
} 
compete directly with one another because some non-merging supplier typically makes the lowest or the second lowest bid in the auctions. One goal of this paper is to clarify the competitive effects of mergers in auction markets.

We examine mergers using an open auction model of procurement. In an open auction, the auction begins at some high price, declines with progressively lower bids, and the contract is awarded to the last bidder in the auction. ${ }^{3}$ An open auction is also known as a descendingprice oral auction. If the costs of the suppliers are private information and stochastically independent, the dominant strategy of a supplier in an open auction is to continue bidding as long as the price is above his cost. When the costs are private and independent, an open auction is strategically equivalent to a second-price auction. In a second-price auction, the suppliers submit sealed bids to the buyer, and the buyer purchases the input from the lowest bidder at a price equal to the second lowest bid. The dominant strategy of a supplier in a sealed-bid second-price auction is to submit a bid equal to its cost. This dominant strategy makes open auctions and second-price auctions much more tractable than sealed-bid firstprice auctions when the suppliers are asymmetric with different cost distributions. ${ }^{4}$ This tractability is particularly important if the buyer is also setting a reserve price. However, the results of our analysis will provide some insights into mergers in first-price auction markets. ${ }^{5}$

Our model has some advantages for merger analysis relative to other models in the literature. First, we derive a convenient family of cost distributions from fundamental properties. This family has a parameter with a natural interpretation as the capacity of the suppliers and is directly related to the market share of suppliers in equilibrium. Second, we define a general measure of increased concentration in terms of the capacity profiles of the suppliers. This measure includes mergers as a trivial case. Third, we allow the buyer to employ a reserve price and set it optimally in response to different capacity profiles. The optimal reserve price allows the buyer to moderate the price effects of increased concentration. Fourth, we obtain a general result that increased concentration reduces the welfare of the buyer, even after an optimal reduction in the reserve price. With this result, we can compare a variety of the possible mergers that might arise from a given capacity profile. Fifth, we find that the optimal reserve price can undermine the incentive for mergers from various concentrated

\footnotetext{
${ }^{3}$ While we use the terms open auction and second-price auction to describe the institution used by the buyer, our results apply to any market institution where the lowest cost supplier sells the input.

${ }^{4}$ General treatments of asymmetric first-price auctions can be found in Maskin and Riley (2000), Lebrun (1999), and Waehrer (1997).

${ }^{5}$ Tschantz, Crooke, and Froeb (2000) find that the competitive effects of mergers is similar in first-price and second-price auctions. The open auction model might also be an appropriate representation of a market even though the buyers do not employ a strict descending-price oral auction. For example, buyers might invite an initial sealed bid from the suppliers, suggesting a first-price auction. However, if the buyers subsequently use the low bids to "whipsaw" other suppliers into lowering their bids, an open auction model seems more appropriate.
} 
capacity profiles. This finding contrasts sharply with the results from the Nash-Cournot models. Finally, we compare the incentive to merge in this model to the incentive in an optimal auction.

The paper is organized as follows. In Section 1, we define the auction and characterize the family of cost distributions. In Section 2, we solve for the expected market shares of the suppliers, the expected price paid by the buyer, and the expected profit of the buyer. In Section 3, we define the measure of industry concentration. In Section 4, we provide the general result that increased concentration reduces the expected profit of the buyer. In Section 5, we examine the incentive to merge and the effects of mergers on total welfare and the profits of non-merging suppliers. In Section 6, we discuss the incentive to merge in an optimal auction. Finally, Section 7 concludes with a sample simulation of the merger effects on the buyer.

\section{The MODEL}

We assume that a buyer requests bids from $n$ suppliers who can provide an input necessary for production of a final good. The buyer employs an open auction to select the winning supplier. More generally, the market would be composed of a series of such auctions by a number of buyers.

The buyer has a value $c_{0}$ for the input where $c_{0}$ is known to the buyer and to all of the suppliers. We interpret $c_{0}$ as the buyer's cost of producing the input internally or as the cost of obtaining the input from a source other than the $n$ suppliers. Alternatively, when there are no sources for the input other than the $n$ suppliers, $c_{0}$ should be interpreted as the net revenue from using the input. ${ }^{6}$ The buyer would clearly reject a bid to supply the input at a price above $c_{0}$. However, we will also consider cases in which the buyer can reject bids below $c_{0}$ by committing to a reserve price $r<c_{0}{ }^{7}$ Note that if the buyer cannot commit to a reserve price less than $c_{0}$, we will simply assume that $r=c_{0}$.

Let $N=\{1, \ldots, n\}$ denote the set of suppliers bidding in the auction. We assume that the $i$ th supplier has a capacity parameter $t_{i}$ and draws his cost $c_{i}$ of producing the input from the distribution $G\left(\cdot \mid t_{i}\right)$ with a support of $[\underline{c}, \bar{c}]$ common to all suppliers. Denote the profile of capacities for the suppliers as $\mathbf{t}=\left(t_{1}, \ldots, t_{n}\right)$ and define $\hat{t}=\sum_{i=1}^{n} t_{i}$ as the total industry capacity. We assume that the costs of the suppliers are independently distributed. ${ }^{8}$

\footnotetext{
${ }^{6}$ Thus, $c_{0}$ is the opportunity cost of not purchasing the input from one of the $n$ suppliers. The buyer either incurs $c_{0}$ in internal costs or sacrifices $c_{0}$ in net revenues.

${ }^{7}$ We assume that the buyer uses a single reserve price that applies equally to all of the suppliers. When the suppliers have different cost distributions, the buyer may be able to gain by setting different reserve prices for the different suppliers. In Section 6, we consider an optimal auction which allows for different reserve prices for different suppliers.

${ }^{8}$ This independence assumption would be violated if the costs of suppliers depended on the uncertain future prices of raw materials and if each supplier received a correlated signal about those future prices. Our
} 
Furthermore, each supplier obtains his cost of production prior to submitting a bid to the buyer and need not incur this cost unless he wins the auction to supply the input. Finally we assume that the buyer's value $c_{0}$ is greater than $\underline{c}$.

In order to examine the effects of mergers and concentration, we need to define the size, scale, or capacity of the suppliers in terms of their differing cost distributions. As a benchmark case, we assume three properties on the cost distributions. First, we assume that there are no externalities across the cost distributions of different suppliers. Second, we assume that capacity is homogeneous. Third, we assume that there are no economies or diseconomies of scale. These three properties can be formally expressed as follows:

Property 1 (No Externalities): The cost distribution of each supplier depends only on its own capacity and is independent of the capacities of other suppliers.

Property 2 (Homogeneity): If two suppliers have the same capacity, then they also have the same cost distribution.

Property 3 (Constant Returns): The probability distribution of the lowest cost draw of the suppliers depends only on total industry capacity. In particular, it does not depend on the number of suppliers or how total industry capacity is distributed among the suppliers. That is, for any two capacity profiles $\mathbf{t}=\left(t_{1}, \ldots t_{n}\right)$ and $\mathbf{s}=\left(s_{1}, \ldots s_{m}\right)$ such that $\hat{t}=\hat{s}$ and for any $c \in[\underline{c}, \bar{c}]$,

$$
1-\prod_{i=1}^{n}\left[1-G\left(c \mid t_{i}\right)\right]=1-\prod_{i=1}^{m}\left[1-G\left(c \mid s_{i}\right)\right] .
$$

For a merger of two suppliers with capacities $t_{i}$ and $t_{j}$, the capacity of the merged supplier is $t_{m}=t_{i}+t_{j}$. Thus, constant returns implies that the cost distribution of this merged supplier is the distribution of the minimum of the two cost draws from the original suppliers. ${ }^{9}$ That is, $G\left(c \mid t_{i}+t_{j}\right)=1-\left[1-G\left(c \mid t_{i}\right)\right]\left[1-G\left(c \mid t_{j}\right)\right] .{ }^{10}$ The following result characterizes Properties 1, 2, and 3 in terms of the functional form of the cost distribution.

Theorem 1. Properties 1, 2, and 3 are satisfied if and only if there exists a distribution function $F$ with a support of $[\underline{c}, \bar{c}]$ such that for $c \in[\underline{c}, \bar{c}), \quad G\left(c \mid t_{i}\right)=1-[1-F(c)]^{t_{i}}$.

model assumes that no such correlation exists. Thus, if raw material prices are relevant, we assume that these costs are known and common to the bids of all suppliers. Any remaining uncertainty about the costs of each supplier would depend only on characteristics unique to that supplier.

${ }^{9}$ The quadratic cost model in Perry and Porter (1985) possesses this property of constant returns. Define $x(c, s)$ as the quantity of output that can be produced at or below a marginal cost of $c$, given the capacity $s$. The quadratic cost function implies that $x\left(c, s_{i}+s_{j}\right)=x\left(c, s_{i}\right)+x\left(c, s_{j}\right)$.

${ }^{10}$ Brannman and Froeb (1997), Waehrer (1997), Dalkir, Logan, and Masson (2000), and Froeb, Tschantz, and Crooke (1998) also model mergers in this way. This type of merger is equivalent to efficient collusion as discussed by Mailath and Zemsky (1991) and McAfee and McMillan (1992). 
The proof of Theorem 1 is in the Appendix. For the remainder of our analysis, we assume Properties 1, 2, and 3 are satisfied and thus, make use of the family of cost distributions provided by Theorem $1 .^{11}$

It is possible to interpret the capacity parameter $t_{i}$ as the number of draws from a cost distribution $F$, where the supplier uses the lowest draw for its cost of producing the input. As such, $G\left(\cdot \mid t_{i}\right)$ is the distribution function of the order statistic for the lowest cost of $t_{i}$ independent draws from $F .{ }^{12}$ Similarly, the capacity parameter $t_{i}$ can be interpreted as the number of plants from which the supplier could produce the input, each plant having a cost distribution $F$. This is the sense in which the parameter $t_{i}$ measures the size, scale, or capacity of a supplier. Thus, we will refer to $t_{i}$ as the capacity of the $i$ th supplier.

\section{Market Equilibrium and Expected Price}

In the equilibrium of the open auction market that we consider, the lowest cost supplier wins at a price equal to the lower of the reserve price and the second lowest cost. When setting the reserve price, the buyer knows the capacities of the suppliers but does not know their actual cost realizations. For any given auction, there would be at most one winning supplier. Hence, in order to assess the industry performance, we examine the expected market share. The expected market share of a supplier is its probability of winning a given auction conditional on some supplier winning the auction.

Define the random variable $z$ as the lowest cost of the $n$ suppliers, $z=\min \left\{c_{1}, \ldots, c_{n}\right\}$. By Property 3, the probability distribution of $z$ is $\operatorname{Pr}\{z \leqslant c\}=G(c \mid \hat{t})$. We can now state the following lemma.

Lemma 1. Suppose the capacity profile is t. (a) The ex ante expected market share of the $i$ th supplier is the ratio of its capacity and the total industry capacity, $t_{i} / \hat{t}$. (b) The ex ante expected profit of the ith supplier is $\Pi^{i}(r \mid \mathbf{t})=\int_{\underline{c}}^{r}\left\{\left[1-F\left(c_{i}\right)\right]^{\hat{t}-t_{i}}-\left[1-F\left(c_{i}\right)\right]^{\hat{t}}\right\} d c_{i}$.

The proof of Lemma 1 is in the Appendix. The expected market share of each supplier is equivalent to its capacity share and independent of the reserve price. Holding the reserve price constant, a supplier's expected profit depends only on its capacity $t_{i}$ and the industry

\footnotetext{
${ }^{11}$ Waehrer (1997) makes use of this family of distributions. This family generalizes the cost structure assumed by Marshall, et.al. (1994) and Dalkir, Logan, and Masson (2000) in that those models assume that $F$ is the uniform distribution. Brannman and Froeb (1997) and Froeb, Tschantz, and Crooke (1998) employ the extreme value distribution for which a parameter similar to $t$ can be defined.

${ }^{12}$ Piccione and Tan (1996) obtain the same functional form from a property which they call "complete stochastic ordering". In Piccione and Tan (1996) and an earlier paper Tan (1992), the exponent $t$ is interpreted as research and development activity. Both papers examine the symmetric equilibrium in research and development expenditures by firms competing in an auction for a production contract. Burguet and Perry (2000) interpret this exponent $t$ as capacity and examine the incentives of suppliers to invest prior to a first-price auction with bribery.
} 
capacity $\hat{t}$. Expected profit does not depend on how the capacity $\hat{t}-t_{i}$ is distributed among the other suppliers. Thus, a merger does not alter the expected profits of the non-merging suppliers or potential entrants when the buyer's reserve price remains unchanged. ${ }^{13}$ For $r \in(\underline{c}, \bar{c})$, a supplier's expected profit is clearly increasing in the reserve price. A higher reserve price does not alter a supplier's market share, but it does increase the probability of purchase by the buyer.

It is straightforward to see that in equilibrium the expected lowest cost of producing the input conditional on purchase from a supplier can be written as

$$
E[z \mid z \leqslant r]=\frac{\int_{\underline{c}}^{r} c d G(c \mid \hat{t})}{G(r \mid \hat{t})} .
$$

If the reserve price is fixed, then the expected lowest cost depends only on total industry capacity $\hat{t}$ and not on the profile $\mathbf{t}$. The expected price conditional on purchase by the buyer is

$$
p(r \mid \mathbf{t})=E[z \mid z \leqslant r]+\frac{\sum_{i=1}^{n} \Pi^{i}(r \mid \mathbf{t})}{G(r \mid \hat{t})} .
$$

The expected price is equal to the expected lowest cost plus the total expected profits of the suppliers, both conditional on purchase. This expected price is the market price that would be observed and does not include the cost of internal production when the reserve price is not met.

The expected profit of the buyer, $U(r \mid \mathbf{t})$, is the net revenue $R$ from using the input minus the buyer's expected cost $E C(r \mid \mathbf{t}) .{ }^{14}$ The buyer's expectd cost $E C(r \mid \mathbf{t})$ is the internal cost $c_{0}$ when the reserve price is not met plus the expected price when the reserve price is met. Thus, $U(r \mid \mathbf{t})=R-E C(r \mid \mathbf{t})$, where

$$
E C(r \mid \mathbf{t})=c_{0} \operatorname{Pr}\{z>r\}+p(r \mid \mathbf{t}) \operatorname{Pr}\{z \leqslant r\}
$$

If the reserve price is fixed, then the capacity profile enters $p(r \mid \mathbf{t})$ and hence $U(r \mid \mathbf{t})$ only through its effect on the sum of the expected profits of the suppliers. Thus, the effect of a merger on the expected price and the expected profit of the buyer will follow directly from its effect on the total expected profits of the suppliers. On the other hand, if the buyer can optimally set the reserve price, then it will also depend on the capacity profile. As a

\footnotetext{
${ }^{13}$ As Waehrer (1997) points out, this would not be true in a first-price auction. A profitable merger in a first-price auction would increase the expected profit of both non-merging suppliers and potential entrants. Similarly, this is not true in the asymmetric Nash-Cournot model. A merger in that model increases the profits of the non-merging firms because the higher price after the merger induces them to expand output.

${ }^{14}$ If the only source for the input is the $n$ suppliers, then the buyer's value $c_{0}$ is the net revenue $R$ and the expected profit reduces to $U(r \mid \mathbf{t})=\left[c_{0}-p(r \mid \mathbf{t})\right] \operatorname{Pr}\{z \leqslant r\}$.
} 
result, the effects of a merger on the expected price and expected profit of the buyer are more complicated. Section 4 will examine the effect of market concentration and mergers on the buyer. That analysis is based on the definition of industry concentration in the next section.

\section{Definitions of Market Concentration}

In this section, we define a concentration ordering which allows us to compare industry performance for different capacity profiles. This ordering facilitates our examination of the welfare effects of mergers and the incentive for mergers. ${ }^{15}$ Consider two capacity profiles $\mathbf{t}$ and $\mathbf{s}$. The profiles $\mathbf{t}$ and $\mathbf{s}$ are equivalent if $\mathbf{s}$ is simply a rearrangement of the elements of t. For parts of the analysis, it is useful to order the capacities in a profile by size. Thus, we define the index notation $t_{(i)}$ such that $t_{(1)} \geqslant \cdots \geqslant t_{(n)}$. When the suppliers $\{1, \ldots, n\}$ follow their equilibrium strategies, the profiles $\left(t_{1}, \ldots, t_{n}\right)$ and $\left(t_{1}, \ldots, t_{n}, 0\right)$ will result in the same outcomes. Hence, it is always possible to add suppliers with zero capacity to a market without affecting the equilibrium outcome.

An equalizing transfer between two suppliers is a transfer of capacity from one supplier to another such that the absolute difference in capacities between the two suppliers is reduced. Let $\mathbf{t}$ be the pre-transfer capacity profile and $\mathbf{s}$ be the post-transfer capacity profile. More formally, an equalizing transfer of capacity from $\mathbf{t}$ to $\mathbf{s}$ for firms $j, k \in N$ requires that (i) $\left|t_{j}-t_{k}\right|>\left|s_{j}-s_{k}\right|$, (ii) $t_{j}+t_{k}=s_{j}+s_{k}$, and (iii) $t_{i}=s_{i}$, for all $i \in N \backslash\{j, k\}$. In applying this definition, it is important to note that any profile can be arbitrarily reindexed without changing its properties.

Definition 1. Capacity profile $\mathbf{t}$ is more concentrated than $\mathbf{s}$ by the transfer principle $\left(\mathbf{t} \succ_{T} \mathbf{s}\right)$ if and only if $\mathbf{s}$ can be constructed from $\mathbf{t}$ by applying a finite series of equalizing transfers. $^{16}$

By the transfer principle, no profile is less concentrated than one where all suppliers have the same capacity. It will be useful to employ the following equivalent definition.

DeFinition 2. Capacity profile $\mathbf{t}$ is more concentrated than $\mathbf{s}$ by second-order dominance $\left(\mathbf{t} \succ_{S D} \mathbf{s}\right)$ if and only if for all $m=1, \ldots, n, \sum_{i=m}^{n} t_{(i)} \leqslant \sum_{i=m}^{n} s_{(i)}$ with the inequality strict for at least one $m .^{17}$

\footnotetext{
${ }^{15}$ Encaoua and Jacquemin (1980) discuss the application of concentration measures to entry and mergers.

${ }^{16}$ The Pigou-Dalton condition holds that inequality should increase when income is transferred from a poorer individual to a richer individual. While stated differently, the Pigou-Dalton transfer condition implies a concentration ordering that is equivalent to the transfer principle.

${ }^{17}$ Shorrocks and Foster (1987) and Foster and Sen (1997) describe a number of concentration or inequality concepts such as generalized Lorenz dominance that are equivalent to second-order dominance (also referred to as second-order stochastic dominance).
} 
In words, $\mathbf{t}$ is more concentrated than $\mathbf{s}$ by second-order dominance if for all $m=1, \ldots, n$, the sum of the capacities of the $(n-m)$ th smallest suppliers from $\mathbf{t}$ is not greater for than the sum of the capacities of the $(n-m)$ th smallest suppliers from $\mathbf{s}{ }^{18}$ Second-order dominance is useful for comparing profiles because it may be difficult to identify the equalizing transfers or to prove that no such transfers exist. Finally, we define the Herfindahl-Hirschman Index (HHI), which the Justice Department and the Federal Trade Commission use in the Merger Guidelines. ${ }^{19}$

Definition 3. Capacity profile $\mathbf{t}$ is more concentrated than $\mathbf{s}$ by the HerfindahlHirschman Index $\left(\mathbf{t} \succ_{H} \mathbf{s}\right)$ if and only if $\sum_{i=1}^{n} t_{i}^{2}>\sum_{i=1}^{n} s_{i}^{2}$ and $\hat{t}=\hat{s}$.

A merger clearly results in a more concentrated industry under all three definitions. The following proposition summarizes the relationship between these three definitions.

Proposition 1. For capacity profiles $\mathbf{t}$ and $\mathbf{s}$ such that $\hat{t}=\hat{s}$,

(a) $\mathbf{t} \succ_{\mathrm{T}} \mathbf{s}$ if and only if $\mathbf{t} \succ_{\mathrm{SD}} \mathbf{s}$,

(b) if $\mathbf{t} \succ_{\mathrm{T}} \mathbf{s}$, then $\mathbf{t} \succ_{\mathrm{H}} \mathbf{s}$.

These results are known from the literatures on mergers and income inequality. ${ }^{20}$ Neither the transfer principle nor second-order dominance can compare every pair of capacity profiles even when the total industry capacity is fixed. However, we show in the next section that when two profiles can be ordered, that ordering unambiguously predicts the comparison between the buyer's expected profit and the optimal reserve price for the two profiles. Unlike the transfer principle, HHI generates a complete ordering of capacity profiles. Hence, there are cases where $\mathbf{t} \succ_{H} \mathbf{s}$ but not $\mathbf{t} \succ_{T} \mathbf{s} .^{21}$ However, we will illustrate in the next section that the HHI can generate inconsistant predictions regarding the buyer's expected profit.

\section{The Effects of Concentration and Mergers on the Buyer}

In this section, we examine the effect of industry concentration on the expected price and the expected profit of the buyer. The following lemma states that the total expected profits of the suppliers is higher for capacity profiles that are more concentrated by the transfer principle. This lemma makes the proof for many of the subsequent results follow quite easily.

\footnotetext{
${ }^{18}$ Since it is easier to identify the large firms in an industry, it is common practice to calculate and compare the shares of the largest suppliers. Second-order dominance is equivalent to this alternative approach when the profiles being compared have the same total capacity.

${ }^{19}$ See U.S. Department of Justice/Federal Trade Commission, Horizontal Merger Guidelines, April 2, 1992 (Revised: April 8, 1997).

${ }^{20}$ For example, see Encaoua and Jacquemin (1980) and Shorrocks and Foster (1987).

${ }^{21}$ For example, for $t=(0.77,0.18,0.05)$ and $s=(0.75,0.25,0.0), \mathbf{t} \succ_{H} \mathbf{s}$ but neither $\mathbf{t} \succ_{T} \mathbf{s}$ nor $\mathbf{s} \succ_{T} \mathbf{t}$.
} 
Lemma 2. If $\mathbf{t} \succ_{T} \mathbf{s}$, then for any $r>\underline{c}, \sum_{i=1}^{n} \Pi^{i}(r \mid \mathbf{t})>\sum_{i=1}^{n} \Pi^{i}(r \mid \mathbf{s})$.

The proof of Lemma 2 is in the Appendix. The result follows from the fact that the expected profit of each supplier is concave in its own capacity. The next theorem provides the key results on the effects of increased concentration.

Theorem 2. Suppose $\mathbf{t} \succ_{T} \mathbf{s}$. Then for any $r>\underline{c}$, (a) $U(r \mid \mathbf{t})<U(r \mid \mathbf{s})$ and (b) $p(r \mid \mathbf{t})>$ $p(r \mid \mathbf{s})$. Further suppose that $r_{\mathbf{t}}^{*}$ and $r_{\mathbf{s}}^{*}$ are the reserve prices that maximize the expected profits of the buyer, $U(r \mid \mathbf{t})$ and $U(r \mid \mathbf{s})$. Then (c) $U\left(r_{\mathbf{t}}^{*} \mid \mathbf{t}\right)<U\left(r_{\mathbf{s}}^{*} \mid \mathbf{s}\right)$ and $(d) r_{\mathbf{t}}^{*} \leqslant r_{\mathbf{s}}^{*}$.

Proof. (a) For any given $r>\underline{c}$, we have

$$
U(r \mid \mathbf{t})-U(r \mid \mathbf{s})=\sum_{i=1}^{n} \Pi^{i}(r \mid \mathbf{s})-\sum_{i=1}^{n} \Pi^{i}(r \mid \mathbf{t})<0 .
$$

The inequality follows from Lemma 2 .

(b) Similarly, making use of (3), for any given $r>\underline{c}$, we have

$$
p(r \mid \mathbf{t})-p(r \mid \mathbf{s})=\frac{\sum_{i=1}^{n} \Pi^{i}(r \mid \mathbf{t})-\sum_{i=1}^{n} \Pi^{i}(r \mid \mathbf{s})}{\operatorname{Pr}\{z \leqslant r\}}>0 .
$$

(c) When the reserve price is adjusted to maximize the expected profit of the buyer, $U\left(r_{\mathbf{s}}^{*} \mid \mathbf{s}\right) \geqslant U\left(r_{\mathbf{t}}^{*} \mid \mathbf{s}\right)>U\left(r_{\mathbf{t}}^{*} \mid \mathbf{t}\right)$. The first inequality follows from the fact that $r_{\mathbf{s}}^{*}$ is the profit-maximizing reserve price when the buyer is faced with capacity profile $\mathbf{s}$. The second inequality follows from part (a) of the theorem.

(d) Note that,

$$
\frac{\partial[U(r \mid \mathbf{t})-U(r \mid \mathbf{s})]}{\partial r}=\frac{\partial\left[\sum_{i=1}^{n} \Pi^{i}(r \mid \mathbf{s})-\sum_{i=1}^{n} \Pi^{i}(r \mid \mathbf{t})\right]}{\partial r} \leqslant 0 .
$$

Therefore, $0=\partial U\left(r_{\mathbf{t}}^{*} \mid \mathbf{t}\right) / \partial r \leqslant \partial U\left(r_{\mathbf{t}}^{*} \mid \mathbf{s}\right) / \partial r$. It follows that $r_{\mathbf{t}}^{*} \leqslant r_{\mathbf{s}}^{*}$.

Q.E.D.

Theorem 2 confirms that increases in concentration result in a lower expected profit to the buyer. ${ }^{22}$ In response, the buyer may react by lowering the reserve price, but this will only moderate the decline in his expected profit. An immediate implication of part (a) of Theorem 2 is that for a given number of suppliers, a given total industry capacity, and a given reserve price, the expected price paid by the buyer is minimized when all of the suppliers have the same capacity. ${ }^{23}$

\footnotetext{
${ }^{22}$ Mares (2000b) proves a result corresponding to Theorem 2(a) for an interesting class of common value auctions (his Theorem 3). The model does not allow a reserve price, but the result is made difficult by the fact that larger suppliers bid more aggressively in common value auctions. With their superior information about the common value, larger suppliers reduce the winner's curse correction on their bid. See Mares (2000b) for a general discussion of the effect of bidding rings on the equilibrium bids.

${ }^{23}$ This result is also true in the Nash-Cournot model. See Farrell and Shapiro (1990a) and Werden (1991).
} 
In the remainder of this section, we examine various merger scenarios and consider their impact on concentration, the expected price, the expected profit of the buyer, and the optimal reserve price.

Proposition 2. A merger of two or more suppliers results in a more concentrated market by the transfer principle. Thus, when the reserve price is fixed, a merger results in a lower expected profit to the buyer and a higher expected price. When the reserve price is set optimally in order to maximize the expected profit of the buyer, a merger results in a lower expected profit to the buyer and a lower reserve price. ${ }^{24}$

Proof. Suppose that suppliers $j$ and $k$ merge. For an initial profile s, the resulting capacity profile can be defined as $t_{j}=s_{j}+s_{k}, t_{k}=0$, and $t_{i}=s_{i}$, for all $i \in N \backslash\{j, k\}$. Clearly $\mathbf{t} \succ_{\mathrm{T}} \mathbf{s}$ because reversing the merger is an equalizing transfer. The proof is completed by applying Theorem 2 .

Q.E.D.

With a fixed reserve price, the buyer will receive a lower expected profit because of the higher expected price of the input even though there is no change in the probability of purchase. The higher expected price arises because the price becomes the third highest cost when the two subsidiaries of the merged supplier have the two lowest costs among all the suppliers. By adjusting the reserve price optimally, the buyer may be able to reduce the adverse price effects of the merger, but the envelope theorem still implies that the merger reduces the expected profit of the buyer. ${ }^{25}$ The expected price $p(r \mid \mathbf{t})$ paid to suppliers may not increase (see Example 1 below), but the buyer's expected cost $E C(r \mid \mathbf{t})$ of producing or purchasing the input increases with the merger.

When the buyer can set the reserve price optimally, the buyer possesses some bargaining power as a monopsonist. The optimal reserve price is analogous to a monopsonist choosing the optimal quantity to purchase along an upward sloping supply curve. The internal cost of production of the buyer affects the optimal reserve price. If $c_{0}$ is sufficiently greater than $\bar{c}$, the buyer will find the reserve price too costly to employ. In this situation, a merger will increase the expected price paid by the buyer. However, when $c_{0}$ is lower and the optimal reserve price is below $\bar{c}$, the effect of a merger on the expected price paid by the buyer is ambiguous. The direct effect of higher concentration increases the expected price, but the indirect effect through the reserve price reduces the expected price. The following examples illustrate that the expected price can rise or fall with a merger to monopoly.

\footnotetext{
${ }^{24}$ Graham and Marshall (1987) and Mailath and Zemsky (1991) prove a similar result relating the optimal reserve price. They consider efficient collusion among bidders of different sizes. Both show that the bid-taker sets a more aggressive reserve price as the number of colluding bidders increases.

${ }^{25}$ Thomas (1998) finds some support for the conjecture that the buyer may benefit from the merger of two small firms because they will compete more effectively against the larger firms. Since Proposition 2 does not distinguish between small and large suppliers, this model clearly does not support such a result.
} 
Example 1. Suppose that $\mathbf{s}=\left(\frac{1}{2}, \frac{1}{2}\right)$ and $F$ is the uniform distribution over the unit interval. Now consider the merger of suppliers 1 and 2 . Thus, $\mathbf{t}=(1,0)$. When $c_{0}=1$, the optimal reserve prices are $r_{s}^{*}=5 / 9$ and $r_{t}^{*}=1 / 2$, and the expected prices are $p\left(r_{s}^{*} \mid \mathbf{s}\right)=47 / 90$ and $p\left(r_{t}^{*} \mid \mathbf{t}\right)=1 / 2$. Thus, the expected price falls after the merger.

Example 2. Consider the same assumptions as in Example 1 except that $c_{0}=1.8$. The optimal reserve prices are $r_{s}^{*}=1$ and $r_{t}^{*}=9 / 10$. The expected prices are $p\left(r_{s}^{*} \mid \mathbf{s}\right)=5 / 6$ and $p\left(r_{t}^{*} \mid \mathbf{t}\right)=9 / 10$. Thus, expected price rises to the reserve price after a merger to monopoly.

The fact that the expected price can fall after the merger should not be interpreted as a decrease in the buyer's expected cost of the input. Proposition 2 implies that the buyer's expected cost of producing or purchasing the input increases even though the buyer optimally lowers the reserve price. Thus, in auction markets with a reserve price, one cannot simply examine the market price in order to assess the welfare effects of mergers on buyers. ${ }^{26}$

We can also use Theorem 2 and the transfer principle to compare a variety of mergers between differing suppliers in the industry.

Proposition 3. For any initial capacity profile $\mathbf{t}$, if $t_{j}>t_{k}$ and $t_{g} \geqslant t_{h}$, then a merger between suppliers $j$ and $g$ results in a more concentrated industry by the transfer principle than a merger between suppliers $k$ and $h$.

The proof of Proposition 3 is in the Appendix. Proposition 3 validates the natural intuition that the acquisition of a larger supplier will result in a more concentrated industry than the acquisition of a smaller supplier. Consider four suppliers from a capacity profile t such that $t_{1}>t_{2}>t_{3}>t_{4}$. Proposition 3 clearly implies that a merger of suppliers 1 and 2 results in a more concentrated market than a merger between suppliers 3 and 4, and that a merger of suppliers 1 and 3 results in a more concentrated market than a merger between suppliers 2 and 4 . With the additional condition that $t_{2}+t_{3} \geqslant t_{1}+t_{4}$, second-order dominance implies that a merger of suppliers 2 and 3 results in a more concentrated market than a merger of suppliers 1 and $4 .{ }^{27}$ However, if $t_{1}+t_{4}>t_{2}+t_{3}$, then neither merger results in a market that is more concentrated than the other. Even though the merger of suppliers 1 and 4 creates a larger supplier than the merger of suppliers 2 and 3, it need not result in a lower expected profit for the buyer. ${ }^{28}$

\footnotetext{
${ }^{26}$ In the Nash-Cournot model, mergers unambiguously increase the equilibrium price and reduce consumer welfare.

${ }^{27}$ These two mergers require a comparison of the following two capacity profiles $\left(t_{2}+t_{3}, t_{1}, t_{4}\right)$ and $\left(t_{1}+\right.$ $\left.t_{4}, t_{2}, t_{3}\right)$. The former is more concentrated than the latter under second-order dominance because $t_{3}>t_{4}$ and $t_{2}+t_{3} \geqslant t_{1}+t_{4}$.

${ }^{28}$ For cases where the transfer principle fails to order capacity profiles, it may be tempting to use the HHI. However, in such cases, the HHI cannot be trusted to predict correctly which profile is associated with
} 


\section{The Incentive to Merge and Total Welfare}

The incentive to merge and the welfare effects of a merger depend on how the reserve price is affected. When the reserve price is fixed, we find that total welfare is unchanged and there is always an incentive to merge. However, when the buyer sets the reserve price optimally, total welfare declines as a result of a merger and there may be no incentive to merge. ${ }^{29}$

Total welfare depends directly on the industry capacity and the reserve price but not on the distribution of industry capacity. Total welfare $W(r \mid \mathbf{t})$ can be written as

$$
W(r \mid \mathbf{t})=R-c_{0}+\left(c_{0}-E[z \mid z \leqslant r]\right) \operatorname{Pr}\{z \leqslant r\} .
$$

The first term $\left(R-c_{0}\right)$ is the profit from internal production, and the second term is the expected cost savings from production of the input by the least cost supplier.

Consider a merger between suppliers 1 and 2 . Let $\mathbf{s}$ and $\mathbf{t}$ denote the pre-merger and post-merger capacity profiles. Hence, $t_{1}=s_{1}+s_{2}, t_{2}=0$, and $t_{i}=s_{i}$, for all $i \notin\{1,2\}$. Assuming a fixed reserve price, suppliers 1 and 2 have an incentive to merge if

$$
\Pi^{1}(r \mid \mathbf{t}) \geqslant \Pi^{1}(r \mid \mathbf{s})+\Pi^{2}(r \mid \mathbf{s})
$$

Proposition 4. If the reserve price is unaffected by a merger, then total welfare and the profits of the non-merging suppliers are unaffected by the merger, and there is always an incentive to merge.

Proof. The fact that total welfare and the expected profits of the non-merging suppliers are unaffected by a merger is clear from the expressions for $W(r \mid \mathbf{t})$ and $\Pi^{i}(r \mid \mathbf{t})$.

For the profiles $\mathbf{s}$ and $\mathbf{t}$ defined above, the incentive to merge can be expressed as

$$
\Pi^{1}(r \mid \mathbf{t})-\left[\Pi^{1}(r \mid \mathbf{s})+\Pi^{2}(r \mid \mathbf{s})\right]=\sum_{i=1}^{n} \Pi^{i}(r \mid \mathbf{t})-\sum_{i=1}^{n} \Pi^{i}(r \mid \mathbf{s}) \geqslant 0 .
$$

The equality follows from the fact that $\Pi^{2}(r \mid \mathbf{t})=0$ and $\Pi^{i}(r \mid \mathbf{t})=\Pi^{i}(r \mid \mathbf{s})$, for all $i \notin\{1,2\}$. The inequality follows from Lemma 2 since $\mathbf{t}$ is more concentrated than $\mathbf{s}$ Q.E.D.

lower expected profits for the buyer. It is straightforward to construct examples where the HHI indicates higher concentration but the buyer's expected profit increases. Suppose that $\mathbf{t}=(0.77, .018,0.05)$ and $\mathbf{s}=(0.75, .025,0.0)$. These profiles cannot be ranked by the transfer principle. Note that even though $\mathbf{t} \succ_{H} \mathbf{s}$, a straightforward calculation shows that $U(1 \mid \mathbf{t})>U(1 \mid \mathbf{s})$, when $c_{0}=1$ and $F$ is the uniform distribution on $[0,1]$.

${ }^{29}$ We assume that the buyer sets the reserve price to the level that is optimal given the capacity profile that he faces. However, suppose that the buyer announces to the market that any merger will be met with an adjustment of the reserve price to $\underline{c}$. If the suppliers believe that such a reaction would occur, no merger would take place because any merger would result in zero profits for all suppliers. 
With a fixed reserve price the welfare effects of a merger are straightforward. The probability of purchase remains unchanged, and a merger merely reallocates profits from the buyer to the merged supplier. ${ }^{30}$ There is always an incentive to merge because the merged supplier receives a higher expected price in cases where the two merging suppliers would have had the two lowest costs. The non-merging suppliers are unaffected by the merger because they receive the same expected price when they have the lowest cost.

If the buyer can optimally set the reserve price, the effects of the merger are more interesting. In particular, total welfare will indirectly depend on the distribution of industry capacity across suppliers through its effect on the reserve price.

Proposition 5. If the buyer sets the reserve price optimally given the capacity profile and there is a positive probability that the reserve price is not met, then mergers reduce total welfare and the profits of the non-merging suppliers.

Proof. Note that for $r \in(\underline{c}, \bar{c}), W(r \mid \mathbf{t})$ and $\Pi^{i}(r \mid \mathbf{t})$ are strictly increasing in $r$. From the proof of Lemma 2, it is clear that when the optimal reserve price is less than $\bar{c}$, an increase in concentration will lead to a strict decrease in the optimal reserve price. Q.E.D.

From Theorem 2, the buyer lowers the reserve price after the merger in order to moderate the price effects of the more concentrated industry. Total welfare declines because the lower reserve price reduces the probability of purchase. Even though the market share of a nonmerging supplier remains unchanged, his expected profits decline because the lower reserve price reduces the probability of purchase and may reduce the price received when he wins the auction. ${ }^{31}$

When the reserve price is set optimally, the expected profits of the merged supplier may or may not be greater after the merger. The merged supplier would receive a higher expected price for any fixed reserve price, but the lower reserve price reduces the expected profits of the merged supplier in the same manner as it does for the non-merging suppliers. If the suppliers know that the buyer will optimally reset the reserve price in response to their merger, then they would presumably take this into account in calculating the expected

\footnotetext{
${ }^{30}$ With a fixed reserve price, there is no deadweight loss from a higher expected price. This is a consequence of defining the auction for a single input. Alternatively, if the buyer was purchasing multiple inputs and employed a unit price auction, higher prices would generate a deadweight loss for the buyer. See Spulber (1995). Such a model would be too cumbersome with asymmetric suppliers and an optimal reserve price. Moreover, the optimal reserve price in this model has a similar effect in that it reduces the probability of purchase and generates a reduction in welfare because of the higher probability of internal production.

${ }^{31}$ This result is different from the Nash-Cournot model. In that model, non-merging suppliers obtain higher profits by expanding production in response to the higher market price after the merger. This insight has been used in Antitrust cases to argue that non-merging suppliers cannot successfully challenge a merger because they would have no damages. This auction model provides an explanation of why non-merging suppliers would be harmed by a merger.
} 
profits after a merger. Thus, the negative effects of the lower reserve price can undermine the incentive to merge. Consider the following two examples.

Example 3. Suppose that $\mathbf{s}=\left(1, \frac{1}{2}, \frac{1}{2}\right)$ and $F$ is the uniform distribution over the unit interval. Now consider the merger of suppliers 2 and 3 . Thus, $\mathbf{t}=(1,1,0)$. When $c_{0}=1$, the optimal reserve prices are $r_{s}^{*}=\frac{2}{25}(9-\sqrt{6})$ and $r_{t}^{*}=1 / 2$. The expected profits of the merging suppliers, pre- and post-merger, are $\Pi^{2}\left(r_{s}^{*} \mid \mathbf{s}\right)+\Pi^{3}\left(r_{s}^{*} \mid \mathbf{s}\right) \approx 0.08019$ and $\Pi^{2}\left(r_{t}^{*} \mid \mathbf{t}\right)=1 / 12 \approx 0.08333$. Thus, suppliers 2 and 3 have an incentive to merge.

Example 4. Consider the same assumptions as in Example 3, except suppose that $\mathbf{s}=$ $(1,1)$. Now consider the merger of suppliers 1 and 2 . Thus, $\mathbf{t}=(2,0)$. The optimal reserve prices are $r_{s}^{*}=1 / 2$ and $r_{t}^{*}=1-1 / \sqrt{3}$, and the expected profits of the merging suppliers are $\Pi^{1}\left(r_{s}^{*} \mid \mathbf{s}\right)+\Pi^{2}\left(r_{s}^{*} \mid \mathbf{s}\right) \approx 0.1666$ and $\Pi^{1}\left(r_{t}^{*} \mid \mathbf{t}\right) \approx 0.1535$. Thus, suppliers 1 and 2 have no incentive to merge.

These two examples provide some intuition about the incentive to merge. In Example 4 , the negative effects of the lower reserve price fall completely on the merged supplier since it is the only supplier in the market after the merger. On the other hand, in Example 3, the negative effect of the lower reserve price is shared between the merged supplier and the non-merging supplier.

The surprising result of Example 4 is that merger to monopoly is not profitable for a symmetric duopoly. ${ }^{32}$ The buyer's optimal reserve price facing a monopoly completely undermines the incentive of the duopolists to merge. This suggests that the optimal reserve price of the buyer can undermine a merger wave. The buyer's ability to commit to a reserve price can allow him to maintain a "stable" industry structure in which there would be no incentive for pairwise mergers that would further concentrate the industry. The triopoly profile in Example 3 is not stable, but the duopoly profile in Example 4 is stable.

Table 1 reports the stable profiles that would arise from various initial symmetric profiles. Starting from each initial profile, we exhaust all possible profitable mergers to arrive at the stable profiles. ${ }^{33}$ For Table 1, we assume that $F$ is uniform over the unit interval, that

\footnotetext{
${ }^{32}$ Using a models that are mathematically similar, Thomas (1998) and McAfee (1994) also find that in some cases there is no incentive for merger to monopoly. These results from the auction models are clearly at variance with the symmetric or asymmetric Nash-Cournot models. The symmetric model of Salant, Switzer, and Reynolds (1983) finds an incentive for symmetric duopolists to merge even though there is no incentive for oligopolists to merge. In Perry and Porter (1985), larger firms are more likely to have an incentive to merge, and asymmetric duopolists would always have an incentive to merge. All the gains from a merger to monopoly by duopolists in these models would be internalized.

${ }^{33}$ An effective but inefficient program written in Mathematica checks all possible merger paths reporting the resulting stable profile when all profitable mergers have been exhausted along a particular path. Our program is available upon request. Other profiles may be stable. However, they could not be reached by profitable mergers from the initial symmetric profile.
} 
Table 1:

\begin{tabular}{|c|c|}
\hline $\begin{array}{c}\text { Initial Symmetric } \\
\text { Profile }\end{array}$ & $\begin{array}{l}\text { Resulting Stable Profiles } \\
\text { After all Profitable Megers }\end{array}$ \\
\hline \multicolumn{2}{|l|}{$(\hat{t}=1)$} \\
\hline$(1 / 3,1 / 3,1 / 3)$ & $(1 / 3,1 / 3,1 / 3)$ \\
\hline$(1 / 4, \ldots, 1 / 4)$ & $(1 / 2,1 / 2)$ \\
\hline$(1 / 5, \ldots, 1 / 5)$ & $(2 / 5,2 / 5,1 / 5)$ \\
\hline$(1 / 6, \ldots, 1 / 6)$ & $(1 / 2,1 / 2)$ and $(1 / 3,1 / 3,1 / 3)$ \\
\hline$(1 / 7, \ldots, 1 / 7)$ & $(4 / 7,3 / 7)$ \\
\hline$(1 / 8, \ldots, 1 / 8)$ & $(1 / 2,1 / 2)$ and $(3 / 8,3 / 8,1 / 4)$ \\
\hline$(1 / 9, \ldots, 1 / 9)$ & $(5 / 9,4 / 9)$ and $(1 / 3,1 / 3,1 / 3)$ \\
\hline$(1 / 10, \ldots, 1 / 10)$ & $(1 / 2,1 / 2),(2 / 5,2 / 5,1 / 5)$, and $(2 / 5,3 / 10,3 / 10)$ \\
\hline \multicolumn{2}{|l|}{$(n=6)$} \\
\hline$(1, \ldots, 1)$ & $(6)$ \\
\hline$(1 / 2, \ldots, 1 / 2)$ & $(2,1)$ and $(3 / 2,3 / 2)$ \\
\hline$(1 / 3, \ldots, 1 / 3)$ & $(1,1)$ and $(4 / 3,2 / 3)$ \\
\hline$(1 / 6, \ldots, 1 / 6)$ & $(1 / 2,1 / 2)$ and $(1 / 3,1 / 3,1 / 3)$ \\
\hline$(1 / 12, \ldots, 1 / 12)$ & $(1 / 4,1 / 4)$ and $(1 / 6,1 / 6,1 / 6)$ \\
\hline$(1 / 18, \ldots, 1 / 18)$ & $(1 / 9,1 / 9,1 / 9)$ \\
\hline $\begin{array}{l}\text { Where: } \\
\qquad \begin{array}{l}c_{0}=1 \\
G\left(c \mid t_{i}\right)=1-\end{array}\end{array}$ & $1-c)^{t_{i}}$, for $c \in[0,1]$ \\
\hline
\end{tabular}

the buyer's cost of internal production is $c_{0}=1$, and that the buyer sets the reserve price optimally for each profile.

In the first part of Table 1, the initial symmetric profiles have the same industry capacity of $\hat{t}=1$. Thus, the expected industry cost $(E[z]=1 / 2$, for $\hat{t}=1)$ remains constant for all the initial profiles. Several interesting findings arise. First, small suppliers have an incentive to merge, but large suppliers may not. Second, different stable profiles can arise from the same initial profile as a result of a different sequence of profitable mergers. Third, the reduction in the optimal reserve price is not sufficient to create stable profiles having more than three suppliers. Fourth, the optimal reserve price eliminates the incentive for a large supplier to merge once its capacity is near half of the industry capacity.

One important determinant of stable profiles is the industry capacity which determines the expected industry cost $E[z]$. The second part of Table 1 reports the stable profiles that arise from initial profiles with six symmetric suppliers, but different total industry capacities. When $\hat{t}=6$ and the initial profile is $(1,1,1,1,1,1)$, the industry will merge to monopoly. With a large industry capacity, the expected industry cost $E[z]$ is well below the buyer's 
internal cost $c_{0}(E[z]=1 / 7$, for $\hat{t}=6)$. As a result, the reserve price is costly for the buyer to employ and becomes less effective at eliminating the incentive to merge. However, when industry capacity is smaller, the reserve price prevents merger to monopoly. As in the first part of Table 1, duopoly and triopoly profiles are stable.

A second important determinant for this analysis of stable profiles is the buyer's internal cost of production $c_{0}$. When the internal cost of production $c_{0}$ is less than or equal to highest possible cost $\bar{c}$ for the suppliers, it is always profitable (ex ante) to set a reserve price $r<c_{0} \leqslant \bar{c}$. In such cases, mergers reduce the reserve price and create the possibility of stable profiles. However, if $c_{0}>\bar{c}$, then the optimal reserve price for some or all profiles need not be less than $\bar{c}$. The reserve price is more costly to employ and is less likely to prevent mergers to monopoly.

Example 5. Consider the same assumptions as in Example 4 except suppose that $c_{0}=$ 2. The optimal reserve prices are $r_{s}^{*}=1$ and $r_{t}^{*}=2 / 3$, and the expected profits of the merging suppliers are $\Pi^{1}\left(r_{s}^{*} \mid \mathbf{s}\right)+\Pi^{2}\left(r_{s}^{*} \mid \mathbf{s}\right)=1 / 3$ and $\Pi^{1}\left(r_{t}^{*} \mid \mathbf{t}\right)=28 / 81>1 / 3$. Thus, unlike Example 4, suppliers 1 and 2 have an incentive to merge.

Examples 4 and 5 illustrate that a higher internal cost of production reduces the ability of the buyer to undermine the incentive for duopolists to merge to monopoly.

The results of this section on the incentive to merge provide a clear contrast with the traditional Nash-Cournot model. In this auction model, the incentive to merge is undermined by the optimal reduction in the reserve price. As a result, larger suppliers with greater capacities are less likely to have an incentive to merge because they would bear most of the burden of the reduction in the reserve price. On the other hand, smaller suppliers would typically have an incentive to merge. Thus, concentrated stable industry structures can arise from mergers. In contrast, no such stability exists in the Nash-Cournot model. The incentive to merge is undermined because the non-merging suppliers expand production in response to the higher price or reduction in output by the merged supplier. Thus, larger suppliers are more likely to have an incentive to merge, and smaller suppliers are less likely. If any merger is profitable, then further mergers to monopoly will also be profitable. ${ }^{34}$

\section{Optimal Auctions}

Thus far we have assumed an open auction where the lowest cost supplier is selected to the supply the input given that the reserve price is met. Moreover, we have assumed that

\footnotetext{
${ }^{34}$ Perry and Porter (1985) illustrate this conclusion using a model with two types of suppliers, one twice as large as the other. There may be no incentive for any merger from an initial industry of all small suppliers. However, if the first merger of two small suppliers is profitable, all subsequent such mergers will be profitable. See the discussion of the incentive to merge in Section III (but note that the second set of Inequalities in (20) was accidentally reversed).
} 
the buyer selects a single reserve price that applies all of the suppliers. Myerson (1981) shows that when the suppliers are symmetric and the reserve price is selected to maximize the profits of the buyer, the open auction is optimal in that there is no other purchasing mechanism that will increase the profits of the buyer. However, the open auction is not optimal when the suppliers are asymmetric. An optimal auction would favor suppliers with lower capacities. In particular, a supplier with a lower capacity might be selected to supply the input even when a supplier with higher capacity has a lower cost. The optimal auction may involve different reserve prices for the different suppliers: a lower reserve price for higher capacity suppliers and a higher reserve price for lower capacity suppliers. Optimal auctions are more difficult to design and implement and thus less useful for policy analysis. However, we will briefly describe the implications of optimal auctions for the incentive to merge.

In an optimal auction, smaller suppliers with a given cost have a higher probability of winning than in an open auction with an optimal reserve price. Hence, smaller suppliers have an advantage in an optimal auction that is not present in an open auction. Smaller suppliers will then find it less profitable to merge because they would relinquish the advantage from being small. Similarly, a merger of large suppliers will increase the disadvantage of the merged supplier and thus reduce the incentive to merge. Hence, we generally expect a reduced incentive to merge in an optimal auction. The following example illustrates this intuition.

Example 6. Suppose that $\mathbf{s}=\left(1, \frac{1}{2}, \frac{1}{2}\right), c_{0}=1$, and $F$ is the uniform distribution over the unit interval. Now consider the merger of suppliers 2 and 3. Thus, the post-merger profile is $\mathbf{t}=(1,1,0)$. Assuming that the buyer uses an optimal auction, the expected profits of the merging suppliers, pre- and post-merger, are $\Pi_{o}^{2}\left(r_{s}^{*} \mid \mathbf{s}\right)+\Pi_{o}^{3}\left(r_{s}^{*} \mid \mathbf{s}\right) \approx 0.08985$ and $\Pi_{o}^{2}\left(r_{t}^{*} \mid \mathbf{t}\right)=1 / 12 \approx 0.08333 .{ }^{35}$ Thus, suppliers 2 and 3 have no incentive to merge in an optimal auction.

With the same assumptions as in Example 6, Example 3 illustrates that there is an incentive for suppliers 2 and 3 to merge in an open auction. The intuition regarding the reduced incentive to merge in an optimal auction is only valid for profiles with three or more suppliers.

\section{Conclusions for Antitrust Policy}

We have examined the qualitative effect of mergers or other increases in market concentration. However, in practice it is often desirable to obtain quantitative estimates of the effects of a merger. As with other market models, the effects of a merger can be simulated.

\footnotetext{
${ }^{35}$ These calculations are based on the solutions provided by Myerson (1981).
} 
Table 2:

\begin{tabular}{|c|c|cc|cc|}
\hline \hline \multicolumn{6}{|c|}{$c_{0}=1$} \\
\hline $\mathbf{t}$ & $r^{*}$ & $E C\left(r^{*} \mid \mathbf{t}\right)$ & $\% \Delta$ & $E C\left(c_{0} \mid \mathbf{t}\right)$ & $\% \Delta$ \\
\hline$(1 / 4,1 / 4,1 / 4,1 / 4)$ & 0.590 & 0.726 & & 0.786 & \\
$(1 / 2,1 / 4,1 / 4)$ & 0.572 & 0.730 & $0.6 \%$ & 0.810 & $3.0 \%$ \\
$(1 / 2,1 / 2)$ & 0.556 & 0.735 & $0.6 \%$ & 0.833 & $3.0 \%$ \\
\hline \hline \multicolumn{5}{|c|}{$c_{0}=3 / 4$} \\
\hline $\mathbf{t}$ & $r^{*}$ & $E C\left(r^{*} \mid \mathbf{t}\right)$ & $\% \Delta$ & $E C\left(c_{0} \mid \mathbf{t}\right)$ & $\% \Delta$ \\
\hline$\{1 / 4,1 / 4,1 / 4,1 / 4\}$ & 0.415 & 0.601 & & 0.677 & \\
$\{1 / 2,1 / 4,1 / 4\}$ & 0.407 & 0.602 & $0.3 \%$ & 0.688 & $1.5 \%$ \\
$\{1 / 2,1 / 2\}$ & 0.400 & 0.604 & $0.2 \%$ & 0.698 & $1.5 \%$ \\
\hline \hline Where: & \multicolumn{5}{l}{} \\
$G\left(c \mid t_{i}\right)=1-(1-c)^{t_{i}}$, for $c \in[0,1]$ & & \\
\hline \hline
\end{tabular}

Merger simulation of auction models requires a calibration of the cost distributions for the suppliers. Theorem 1 provides a justification for using a convenient functional form for $G$. Since the expected market share is $t_{i} / \hat{t}$ from Lemma 1 , the capacity of the $i$ th supplier could be estimated from the observed market share, given the industry capacity $\hat{t}$. The remaining problem is calibrating the other dimensions of $G$, in particular, the range of costs $[\underline{c}, \bar{c}]$, the distribution $F$, the industry capacity $\hat{t}$. Several authors have suggested methods for calibrating distributions for auction models. ${ }^{36}$ Economies of scale could also be incorporated into the simulations by defining $G\left(c \mid t_{i}\right)=1-[1-F(c)]^{\phi\left(t_{i}\right)}$ where $\phi$ is a strictly convex function. Finally, if the buyer sets an optimal reserve price below $\bar{c}$, then simulation would require an estimate for $c_{0}$.

Table 2 illustrates how a simulation could quantify the effects of a merger on a buyer. For the sample simulation, we assume that $F$ is uniform on the unit interval and that $\hat{t}=1$. We then examine the effects of profitable mergers beginning from an initial profile of $(1 / 4,1 / 4,1 / 4,1 / 4)$. In order to illustrate the importance of the internal cost of production and the reserve price, Table 2 reports the effects of each merger for two different values of $c_{0}$ (1 and 3/4), with and without an optimal reserve price. The effect of the merger can be measured by the percentage increase in the buyer's expected cost.

As demonstrated by Theorem 2, the optimal reserve price declines with each merger, and the buyer's expected cost increases. Table 2 illustrates that the percentage increase in the buyer's expected cost is much smaller with the optimal reserve price than without. This

\footnotetext{
${ }^{36}$ See Dalkir, Logan, and Masson (2000), Froeb, Tschantz, and Crooke (1999), and Tschantz, Crooke, and Froeb (2000).
} 
moderating effect arises both from the use of the reserve price and from the reduction in the reserve price after the merger. Thus, the reserve price provides significant protection for the buyer when his internal cost of production is near or within the range of costs for the suppliers.

When the buyer's internal cost of production is lower, the optimal reserve price is also lower, and the buyer's expected cost is lower. If the internal cost of production is less than the upper bound on the costs of the suppliers, then the buyer becomes a competitor in the provision of the input. This also provides an additional element of protection for the buyer. In sum, the buyer's internal cost of production and the optimal reserve price are important moderating factors on the adverse competitive effects of a merger. 


\section{APPENDIX}

Proof of Theorem 1. (If): It is easy to verify that the distribution function as defined in the theorem satisfies P1, P2, and P3.

(Only if): For arbitrary $t_{1}, t_{2} \geqslant 0$, define the profiles $\mathbf{t}=\left(t_{1}, t_{2}\right)$ and $\mathbf{s}=\left(t_{1}+t_{2}\right)$. Note $\mathbf{s}$ contains only one supplier with a capacity of $t_{1}+t_{2}$. Substituting these profiles into (1), Properties 1,2 , and 3 imply that for all $t_{1}, t_{2} \geqslant 0$ and all $c \in[\underline{c}, \bar{c}]$,

$$
G\left(c \mid t_{1}+t_{2}\right)=1-\left[1-G\left(c \mid t_{1}\right)\right]\left[1-G\left(c \mid t_{2}\right)\right]
$$

First we prove that for any $c \in[\underline{c}, \bar{c}], G(c \mid \cdot)$ is nondecreasing in its second argument. For $t_{i}>t_{j} \geqslant 0$,

$$
\begin{aligned}
G\left(c \mid t_{i}\right)-G\left(c \mid t_{j}\right) & =1-\left[1-G\left(c \mid t_{i}-t_{j}\right)\right]\left[1-G\left(c \mid t_{j}\right)\right]-G\left(c \mid t_{j}\right) \\
& =G\left(c \mid t_{i}-t_{j}\right)\left[1-G\left(c \mid t_{j}\right)\right] \geqslant 0 .
\end{aligned}
$$

The first equality follows from (6) since $G\left(c \mid t_{i}\right)=1-\left[1-G\left(c \mid t_{i}-t_{j}\right)\right]\left[1-G\left(c \mid t_{j}\right)\right]$.

In order to show that $G\left(c \mid t_{i}\right)$ is differentiable in its second argument for all $t_{i}>0$, choose an arbitrary $\left(c, t_{i}\right) \in[\underline{c}, \bar{c}] \times \mathbb{R}_{++}$. There exists a $t_{k}>t_{i}$ such that $G(c \mid \cdot)$ is differentiable in its second argument at $t_{k}$ because, being monotonic, $G(c \mid \cdot)$ is differentiable almost everywhere. Define $t_{j}>0$ such that $t_{k}=t_{i}+t_{j}$. By choice of $t_{k}, G(c \mid \cdot)$ is differentiable in its second argument at $t_{k}$, and thus, $G\left(c \mid t_{i}+t_{j}\right)$ is differentiable in $t_{i}$. With $t_{i}$ and $t_{j}$ replacing $t_{1}$ and $t_{2}$, equality (6) holds for all $t_{i}, t_{j} \geqslant 0$. Therefore, that the left-hand side is differentiable in $t_{i}$ implies that the right-hand side and thus, $G\left(c \mid t_{i}\right)$ is differentiable in $t_{i}$.

Replacing $t_{1}$ with $t_{i}$ and $t_{2}$ with $t_{j}$ and differentiating (6) and noting that $\partial G\left(c \mid t_{i}+\right.$ $\left.t_{j}\right) / \partial t_{i}=\partial G\left(c \mid t_{i}+t_{j}\right) / \partial t_{j}$, we have, for any $c \in[\underline{c}, \bar{c})$,

$$
\frac{\partial G\left(c \mid t_{i}\right) / \partial t_{i}}{\left[1-G\left(c \mid t_{i}\right)\right]}=\frac{\partial G\left(c \mid t_{j}\right) / \partial t_{j}}{\left[1-G\left(c \mid t_{j}\right)\right]}
$$

By Property 1 , the left-hand side of the equality does not depend on $t_{j}$. Therefore, since the equality holds for all $t_{i}, t_{j} \geqslant 0$, the ratio $\left[\partial G\left(c \mid t_{i}\right) / \partial t_{i}\right] /\left[1-G\left(c \mid t_{i}\right)\right]$ is constant with respect to $t_{i}$. Moreover, $G(c \mid \cdot)$ must satisfy a differential equation of the form $\partial G\left(c \mid t_{i}\right) / \partial t_{i}=$ $\left[1-G\left(c \mid t_{i}\right)\right] k(c)$ where $k(c)$ can depend on $c$ but not on $t_{i}$. This is a first-order linear differential equation which has a unique solution. In fact, the solution can be written as $G\left(c \mid t_{i}\right)=1-\sigma(c)[1-F(c)]^{t_{i}}$ where $F(c)=1-e^{-k(c)}$ and $\sigma(c)$ is a constant term with respect to $t_{i}$. The fact that $G(c \mid 0)=0$ for all $c \in[\underline{c}, \bar{c})$ follows immediately from (6). Therefore, $\sigma(c)=1$. Since $G$ is a distribution function with a support of $[\underline{c}, \bar{c}], F$ must also be a distribution function with a support of $[\underline{c}, \bar{c}]$.

Q.E.D. 
Proof of Lemma 1. (a) The probability that all of the $i$ th supplier's rivals have costs higher than $c_{i}$ is $\prod_{j \neq i}\left[1-F\left(c_{i}\right)\right]^{t_{j}}=\left[1-F\left(c_{i}\right)\right]^{\hat{t}-t_{i}}$. Since each supplier submits a bid equal to its cost and the lowest cost supplier wins, the $i$ th supplier's ex ante probability of winning the auction is equal to the probability that all of the other suppliers have a higher cost and its cost is below the reserve price. The $i$ th supplier's ex ante probability of winning is equal to

$$
\begin{aligned}
\int_{\underline{c}}^{r}\left[1-F\left(c_{i}\right)\right]^{\hat{t}-t_{i}} t_{i}\left[1-F\left(c_{i}\right)\right]^{t_{i}-1} f\left(c_{i}\right) d c_{i} & =t_{i} \int_{\underline{c}}^{r}\left[1-F\left(c_{i}\right)\right]^{\hat{t}-1} f\left(c_{i}\right) d c_{i} \\
& =\frac{t_{i}}{\hat{t}}\left(1-[1-F(r)]^{\hat{t}}\right) .
\end{aligned}
$$

Hence, the $i$ th supplier's ex ante probability of winning, conditional on some supplier winning, is $\frac{t_{i}}{\hat{t}}\left(1-[1-F(r)]^{\hat{t}}\right)\left(\sum_{j=1}^{n} \frac{t_{j}}{\hat{t}}\left(1-[1-F(r)]^{\hat{t}}\right)\right)^{-1}=\frac{t_{i}}{\hat{t}}$.

(b) Let $\pi^{i}\left(c_{i}, r \mid \mathbf{t}\right)$ denote the $i$ th supplier's expected profit in equilibrium when he has $\operatorname{costs} c_{i}$, the reserve price is $r$, and the capacity profile is $\mathbf{t}$.

$$
\begin{aligned}
\pi^{i}\left(c_{i}, r \mid \mathbf{t}\right) & =\left(\int_{c_{i}}^{r}\left[\xi-c_{i}\right]\left(\hat{t}-t_{i}\right)[1-F(\xi)]^{\hat{t}-t_{i}-1} f(\xi) d \xi+\left(r-c_{i}\right)[1-F(r)]^{\hat{t}-t_{i}}\right) \cdot 1_{\left\{c_{i} \leqslant r\right\}} \\
& =\int_{c_{i}}^{r}[1-F(\xi)]^{\hat{t}-t_{i}} d \xi \cdot 1_{\left\{c_{i} \leqslant r\right\}} \quad \text { (using integration by parts). }
\end{aligned}
$$

The indicator function, $1_{\left\{c_{i} \leqslant r\right\}}$, is equal to one when the condition within the braces is true and zero otherwise. The $i$ th supplier's ex ante expected profit is $\Pi^{i}(r \mid \mathbf{t})=E\left[\pi^{i}\left(c_{i}, r \mid \mathbf{t}\right)\right]$ where the expectation is taken over his cost.

$$
\begin{aligned}
\Pi^{i}(r \mid \mathbf{t}) & =\int_{\underline{c}}^{r} \int_{c_{i}}^{r}[1-F(\xi)]^{\hat{t}-t_{i}} t_{i}\left[1-F\left(c_{i}\right)\right]^{t_{i}-1} f\left(c_{i}\right) d \xi d c_{i} \\
& =\int_{\underline{c}}^{r}\left\{\left[1-F\left(c_{i}\right)\right]^{\hat{t}-t_{i}}-\left[1-F\left(c_{i}\right)\right]^{\hat{t}}\right\} d c_{i} \quad \text { (using integration by parts). }
\end{aligned}
$$

Q.E.D.

Proof of Lemma 2. To prove the lemma for arbitrary $\mathbf{t}$ and $\mathbf{s}$ such that $\mathbf{t} \succ_{\mathrm{T}} \mathbf{s}$, it is sufficient to prove the lemma for $\mathbf{t}$ and $\mathbf{s}$ that differ by a single equalizing transfer. Hence, define $\mathbf{t}$ and $\mathbf{s}$ such that for some $j, k \in N, t_{j}>s_{j} \geqslant s_{k}>t_{k}, t_{j}+t_{k}=s_{j}+s_{k}$, and $t_{i}=s_{i}$, for all $i \in N \backslash\{j, k\}$. Let $\alpha=t_{j}-s_{j}$. Define the function $\widetilde{\Pi}$ as $\widetilde{\Pi}\left(r, t_{i}, \widehat{t}\right)=$ $\int_{\underline{c}}^{r}\left\{[1-F(c)]^{\hat{t}-t_{i}}-[1-F(c)]^{\hat{t}}\right\} d c$. It is straightforward to verify that $\widetilde{\Pi}$ is strictly convex in its second argument. Thus,

$\sum_{i=1}^{n} \Pi^{i}(r \mid \mathbf{t})-\sum_{i=1}^{n} \Pi^{i}(r \mid \mathbf{s})=\widetilde{\Pi}\left(r, t_{j}, \widehat{t}\right)+\widetilde{\Pi}\left(r, t_{k}, \widehat{t}\right)-\widetilde{\Pi}\left(r, s_{j}, \widehat{t}\right)-\widetilde{\Pi}\left(r, s_{k}, \widehat{t}\right)$ 


$$
=\left[\widetilde{\Pi}\left(r, t_{j}, \widehat{t}\right)-\widetilde{\Pi}\left(r, t_{j}-\alpha, \widehat{t}\right)\right]-\left[\widetilde{\Pi}\left(r, s_{k}, \widehat{t}\right)-\widetilde{\Pi}\left(r, s_{k}-\alpha, \widehat{t}\right)\right]>0
$$

The first and second equalities follow after substituting, canceling, and rearranging terms. The inequality follows from the strict convexity of $\widetilde{\Pi}$ since $t_{j}>s_{k}$. Q.E.D.

Proof of Proposition 3. We first show that for any $j, k, g \in N$ such that $t_{j}>t_{k}$, a merger between suppliers $j$ and $g$ results in a more concentrated market than a merger between suppliers $k$ and $g$. Let $\mathbf{s}^{1}$ denote the capacity profile after the merger of $j$ and $g$, and let $\mathbf{s}^{2}$ denote the capacity profile after the merger of $k$ and $g$. Specifically, $s_{g}^{1}=s_{g}^{2}=0$, $s_{j}^{1}=t_{g}+t_{j}, s_{k}^{2}=t_{g}+t_{k}, s_{k}^{1}=t_{k}, s_{j}^{2}=t_{j}$, and for all $i \in N \backslash\{j, k\}, s_{i}^{1}=s_{i}^{2}=t_{i}$. Note that $\sum_{i=1}^{n} s_{i}^{1}=\sum_{i=1}^{n} s_{i}^{2}$. Therefore, $\mathbf{s}^{1} \succ_{\mathrm{T}} \mathbf{s}^{2}$ if $\left|s_{j}^{1}-s_{k}^{1}\right|>\left|s_{j}^{2}-s_{k}^{2}\right|$. This inequality is equivalent to $\left|t_{g}+t_{j}-t_{k}\right|>\left|t_{g}+t_{k}-t_{j}\right|$ which follows from $t_{j}>t_{k}$.

For any $j, k, g, h \in N$, such that $t_{j}>t_{k}$ and $t_{g} \geqslant t_{h}$, consider the capacity profiles that result from the mergers of suppliers $j$ and $g$ and suppliers $k$ and $h$. For the case where $t_{g}=t_{h}$, the proof is complete. Now suppose $t_{g}>t_{h}$. With an initial profile $\mathbf{t}$, let $\mathbf{s}^{3}$ denote the capacity profile after the merger of firms $k$ and $h$. Note that $\mathbf{s}^{2} \succ_{\mathrm{T}} \mathbf{s}^{3}$ follows from the result established in the first part of the proof. Therefore, by transitivity $\mathbf{s}^{1} \succ_{\mathrm{T}} \mathbf{s}^{3}$. Q.E.D. 


\section{REFERENCES}

Brannman, Lance and Luke M. Froeb (2000) "Mergers, Cartels, Set-Asides and Bidding Preferences in Asymmetric Second-Price Auctions," Review of Economics and Statistics 82(2):283-290.

Burguet, Roberto and Martin K. Perry (2000) "Bribery and Favoritism by Auctioneers in Sealed Bid Auctions," mimeo, Department of Economics, Rutgers University.

Dalkir, Serdar, John W. Logan, and Robert T. Masson (2000) "Mergers in Noncooperative Auction Markets: The Effects of Mergers on Prices and Efficiency," International Journal of Industrial Organization 18:383-413.

DeBrock, Larry M. and James L. Smith (1983) "Joint Bidding, Information Pooling, and the Performance of Petroleum Lease Auctions," Bell Journal of Economics 14:395404.

Deneckere, Raymond and Carl Davidson (1985) "Incentives to Form Coalitions with Bertrand Competition," Rand Journal of Economics 16(4):473-486.

Encaoua, David and Alexis Jacquemin (1980) "Degree of Monopoly, Indices of Concentration and Threat of Entry," International Economic Review 21(1):87-105.

Farrell, Joseph and Carl Shapiro (1990a) "Asset Ownership and Market Structure in Oligopoly," Rand Journal of Economics 21(2): 275-292.

Farrell, Joseph and Carl Shapiro (1990b) "Horizontal Mergers: An Equilibrium Analysis," American Economic Review 80(1):107-126.

Foster, James E. and Amartya Sen (1997) "On Economic Inequality: After a Quarter Century," in On Economic Inequality by Amartya Sen, Enlarged Edition, Oxford: Clarendon Press, 1997.

Froeb, Luke, Steven Tschantz, and Philip Crooke (1998) "Mergers Among Asymmetric Bidders: A Logit Second-Price Auction Model," mimeo, Vanderbilt University.

Graham, Daniel A. and Robert C. Marshall (1987) "Collusive Bidder Behavior at SingleObject Second-Price and English Auctions," Journal of Political Economy 95(6):12171239.

Lebrun, Bernard (1999) "First Price Auction in the Asymmetric $N$ Bidder Case," International Economic Review 40(1):97-110. 
Mailath, George J. and Peter Zemsky (1991) "Collusion in Second Price Auctions with Heterogeneous Bidders," Games and Economic Behavior 3:467-486.

Mares, Vlad (2000a) "Market Concentration and Prices in Common-Value Auctions," mimeo, Department of Economics, Rutgers University.

Mares, Vlad (2000b) "Asymmetric Mergers in Common-Value Auctions," mimeo, Department of Economics, Rutgers University.

Marshall, Robert C., Michael J. Meurer, Jean-Francois Richard, and Walter Stromquist (1994) "Numerical Analysis of Asymmetric First Price Auctions," Games and Economic Behavior 7:193-220.

Maskin, Eric and John Riley (2000) "Asymmetric Auctions," Review of Economic Studies, 67(3):413-438.

McAfee, R. Preston (1994) "Endogenous Availability, Cartels, and Merger in an Equilibrium Price Dispersion," Journal of Economic Theory 62(1):24-47.

McAfee, R. Preston and John McMillan (1992) "Bidding Rings," American Economic Review 82(3):579-599.

McAfee, R. Preston and Michael A. Williams (1992) "Horizontal Mergers and Antitrust Policy," Journal of Industrial Economics 40(2):181-87.

Myerson, Roger B. (1981) "Optimal Auction Design." Mathematics of Operations Research 6(1):58-73.

Perry, Martin K. and Robert H. Porter (1985) "Oligopoly and the Incentive for Horizontal Merger," American Economic Review 75(1):219-227.

Piccione, Michele and Guofo Tan (1996) "Cost-Reducing Investment, Optimal Procurement and Implementation by Auctions," International Economic Review 37(3):663-685.

Salant, Stephen W., Sheldon Switzer, and Robert J. Reynolds (1983) "Losses from Horizontal Merger: The Effects of an Exogenous Change in Industry Structure on CournotNash Equilibrium," Quarterly Journal of Economics 98(2):185-199.

Shorrocks, Anthony F. and James E. Foster (1987) "Transfer Sensitive Inequality Measures," Review of Economic Studies 54(3):485-497.

Spulber, Daniel F. (1995) "Bertrand Competition When Rivals' Costs Are Unknown," Journal of Industrial Economics, 43:1-11 
Tan, Guofu (1992) "Entry and R \& D in Procurement Contracting," Journal of Economic Theory 58(1):41-60.

Thomas, Charles J. (1998) "The Competitive Effects of Mergers Between Asymmetric Firms," Federal Trade Commission, Working Paper \#220.

Tschantz, Steven, Philip Crooke, and Luke Froeb (2000) "Mergers in Sealed vs. Oral Asymmetric Auctions," International Journal of the Economics of Business 7(2):201213.

Von Ungern-Sternberg, Thomas. (1988) "Cartel Stability in Sealed-Bid Second Price Auctions," Journal of Industrial Economics 36(3):351-358.

Waehrer, Keith (1997) "Asymmetric Private Values Auctions With Application to Joint Bidding and Mergers," International Journal of Industrial Organization 17:437-452.

Werden, Gregory J. (1991) "Horizontal Mergers: Comment," American Economic Review 81(4):1002-1006. 
ENDNOTES 